Research Article

\title{
Genomic and Pathogenic Characteristics of Virulent Newcastle Disease Virus Isolated from Chicken in Live Bird Markets and Backyard Flocks in Kenya
}

\author{
Irene N. Ogali $\mathbb{D}^{1,2}$ Paul O. Okumu, ${ }^{3}$ Erick O. Mungube, ${ }^{2}$ Jacqueline K. Lichoti, ${ }^{4}$ \\ Stephen Ogada, ${ }^{1}$ Grace K. Moraa, ${ }^{1}$ Bernard R. Agwanda, ${ }^{5}$ and Sheila C. Ommeh $\mathbb{D}^{1}$ \\ ${ }^{1}$ Institute of Biotechnology Research, Jomo Kenyatta University of Agriculture and Technology, P.O. Box 62000-00200, \\ Nairobi, Kenya \\ ${ }^{2}$ Veterinary Science Research Institute, Kenya Agriculture and Livestock Research Organization, P.O. Box 32-00902, \\ Kikuyu, Kenya \\ ${ }^{3}$ Department of Veterinary Pathology, Microbiology and Parasitology, University of Nairobi, P.O. Box 29053-00625, Kangemi, \\ Nairobi, Kenya \\ ${ }^{4}$ Directorate of Veterinary Services, State Department of Livestock, Ministry of Agriculture Livestock and Fisheries, \\ P.O. Box Private Bag, Kangemi, Kenya \\ ${ }^{5}$ Department of Zoology, National Museums of Kenya, P.O. Box 40658-00100, Nairobi, Kenya
}

Correspondence should be addressed to Irene N. Ogali; inogali@yahoo.com and Sheila C. Ommeh; sommeh@jkuat.ac.ke

Received 19 February 2020; Accepted 22 July 2020; Published 18 August 2020

Academic Editor: Todd R. Callaway

Copyright ( $) 2020$ Irene N. Ogali et al. This is an open access article distributed under the Creative Commons Attribution License, which permits unrestricted use, distribution, and reproduction in any medium, provided the original work is properly cited.

\begin{abstract}
Newcastle disease (ND) causes significant economic losses in the poultry industry in developing countries. In Kenya, despite rampant annual ND outbreaks, implementation of control strategies is hampered by a lack of adequate knowledge on the circulating and outbreak causing-NDV strains. This study reports the first complete genome sequences of NDV from backyard chicken in Kenya. The results showed that all three isolates are virulent, as assessed by the mean death time (MDT) and intracerebral pathogenicity index (ICPI) in specific antibody negative (SAN) embryonated eggs and 10-day-old chickens, respectively. Also, the polybasic amino acid sequence at the fusion-protein cleavage site had the motif ${ }^{112} \mathrm{RRQKRFV}^{118}$. Histopathological findings in four-week-old SPF chicken challenged with the NDV isolates KE001, KE0811, and KE0698 showed multiple organ involvement at five days after infection with severe effects seen in lymphoid tissues and blood vessels. Analysis of genome sequences obtained from the three isolates showed that they were 15192 base pair (bp) in length and had genomic features consistent with other NDV strains, the functional sites within the coding sequence being highly conserved in the sequence of the three isolates. Amino acid residues and substitutions in the structural proteins of the three isolates were similar to the newly isolated Tanzanian NDV strain (Mbeya/MT15). A similarity matrix showed a high similarity of the isolates to NDV strains of class II genotype V (89-90\%) and subgenotype Vd (95-97\%). Phylogenetic analysis confirmed that the three isolates are closely related to NDV genotype V strains but form a distinct cluster together with NDV strains from the East African countries of Uganda and Tanzania to form the newly characterized subgenotype Vd. Our study provides the first description of the genomic and pathological characteristics of NDV of subgenotype $\mathrm{Vd}$ and lays a baseline in understanding the evolutionary dynamics of NDV and, in particular, Genotype V. This information will be useful in the development of specific markers for detection of viruses of genotype $\mathrm{V}$ and generation of genotype matched vaccines.
\end{abstract}

\section{Introduction}

Newcastle disease (ND) is a highly contagious disease of poultry that is notifiable to the World Organization for Animal Health
(OIE) [1]. In developing countries, ND has a significant economic impact on the poultry sector due to the high morbidity and mortality [2]. Newcastle disease is caused by Newcastle disease virus (NDV), a single-stranded negative-sense RNA 
virus classified in the genus Avulavirus within the family Paramyxoviridae in the Order Mononegavirales [3]. Newcastle disease virus consists of a $15 \mathrm{~kb}$ genome with six open reading frames (ORF) encoding six major structural proteins, namely, nucleoprotein $(\mathrm{NP})$, phosphoprotein $(\mathrm{P})$, matrix protein $(\mathrm{M})$, fusion-protein $(\mathrm{F})$, hemagglutinin neuraminidase $(\mathrm{HN})$, and the RNA-dependent RNA polymerase (L) in the order, $3^{\prime}$-NP-P-MF-HN-L-5 ${ }^{\prime}[4,5]$. Each of these genes is flanked by short extragenic "leader" and "trailer" sequences. Besides, each gene starts with a conserved gene start (GS) and ends with a conserved sequence: gene end (GE) [6].

Newcastle disease virus, although belonging to one serotype, is highly diverse genetically and antigenically. NDV isolates from the world are grouped into two distinct classes (class I or II), based on genome lengths and nucleotide sequences. Class I NDV is less divergent and belongs to a single genotype, whereas class II isolates are more diverse [5]. Based on the analysis of the complete coding region of the fusion gene, class II NDV has been divided into 18 genotypes [7-10]. Newcastle disease virus belonging to class II, which was isolated between the 1930s and 1960s, includes genotypes I, II, III, and IV. These are considered "ancient" and have genome sizes of 15,186 nucleotides (nt) [11]. The currently circulating or "recent" class II genotypes were isolated after the 1960s (V, VI, VII, VIII, and X-XVIII). The latter genotypes have an insertion of six nucleotides into the $5^{\prime}$ noncoding region of the nucleoprotein gene. They, therefore, possess $15192 \mathrm{nt}$ in their genomes [11]. More genetic distance has been observed between "ancient" and the "recent" genotypes [5]. Some of the genotypes are further divided into subgenotypes according to set criteria [7].

The most recent genotypes (XIV-XVIII) have mostly been isolated on the African continent $[8,10,12]$. A study on isolates from Southern Africa as well as from Namibia, Zambia, Zimbabwe, and Mozambique revealed the presence of genotypes VIII and VII $[13,14]$. In Zambia, genotype XIII has also been isolated from poultry [15]. A comparative analysis of APMV-1 sequences from West Africa indicated that the viruses correspond to genotypes I and VI and novel genotype XVIII [16]. Novel genotypes XIV and XVII have also been isolated from Western and Central African countries $[17,18]$. Isolates from Ethiopia were assigned to genotypes VII and VI [19-21]. Isolates from Tanzania were assigned to genotypes I, V, and Via, respectively [22]. Previous studies reported that isolates from Sudan belonged to genotypes VII and VI [23, 24]. A study in Eastern Uganda in 2001 revealed that they belonged to genotype VIa , 2004) [25]. A more recent study in Uganda isolated strains of genotype Vd from poultry in LBMs [26]. It is suggested that increased surveillance on the African Continent may lead to the discovery of more genotypes and subgenotypes [27]. This is thought to be a result of the predominance of the backyard poultry management system which encourages the presence and spread of varied strains of velogenic NDV which threatens commercial poultry enterprises [28].

In Kenya, like other developing countries, the high population of poultry is kept on smallholder backyard farms. Poultry production is important for the livelihoods of many rural households as a source of protein and income and fulfills the sociocultural role. Although Newcastle disease is endemic in Kenya, potential reservoirs of NDV virus exist which are responsible for the annual epidemic outbreaks of ND causing annual epidemic outbreaks that result in high bird mortality, economic losses, and negative impact on the livelihoods of rural populations [1]. The introduction of birds from informal live bird markets (LBMs) poses an important source of introduction of NDV into a flock [29]. Live bird markets may enhance the movement of infected birds and the spread of NDV is rampant in Kenya and may be important in the epidemiology of ND [30]. However, until now, only a few studies have reported the genetic and pathological characteristics of NDV circulating in Kenya. This study for the first time characterized and compared the genomic and pathological characteristics of NDV from live bird markets and backyard poultry farms in Kenya to establish the epidemiological link and role of live bird markets and backyard poultry in the spread of NDV in Kenya.

\section{Materials and Methods}

2.1. Viruses. Cloacal swabs and tissue samples for the study came from chicken showing clinical symptoms of Newcastle disease. These samples were obtained from live bird markets and backyard poultry farms in Kenya during a survey of Newcastle disease virus described by [30]. We inoculated samples from individual birds in three 10-day-old specific antibody negative (SAN) embryonated chicken eggs through the allantoic cavity. Three days after inoculation, the allantoic fluid was harvested and clarified by centrifugation at $4000 \times \mathrm{g}$ for 20 minutes at $4^{\circ} \mathrm{C}$. We used the supernatant to run the hemagglutination inhibition test using specific antisera to the reference strains of NDV. Three NDV isolates designated KE001/2015 (Makueni), KE0811/2016 (Mombasa), and KE0698/2016 (Malaba) showed high HA titer $\left(>2^{4}\right)$. The allantoic fluid of the three isolates was aliquoted for use in pathogenicity tests and genome sequencing and characterization.

2.2. Study Clearance and Permits of Compliance. The Kenya Agricultural and Livestock Research Organization (KALRO), Veterinary Science Research Institute- (VSRI-) Institutional Animal Care and Use Committee (IACUC), approved poultry handling procedures used in the study (protocol number: KALRO/VSRI/IACUC/1/23082017) according to the appropriate animal guidelines [31]. We carried out the study in the biosafety level II isolation facility at KALRO-VSRI.

2.3. Biological Characterization of the NDV Isolates. The pathogenic potential of the three NDV isolates was evaluated using a standard assay to determine mean death time (MDT) in 10-day-old specific antibody negative (SAN) embryonated eggs and the intracerebral pathogenicity index (ICPI) in 1-day-old chicks [1]. Briefly, ICPI was performed in 1day-old chicks by inoculation of $50 \mu \mathrm{L}$ of allantoic fluid diluted 10-fold in PBS without antibiotics as recommended [1]. We calculated the intracerebral pathogenicity index as 
the mean of each observation for each bird. We observed the birds every 24 hours for 1 week. At each observation, the birds were scored 0 if normal, 1 if sick, and 2 if dead. We humanely sacrificed birds that were too ill and unable to eat by cervical dislocation and scored them as dead in the next observation. We gave dead birds a score of two at each of the remaining observations.

We considered Newcastle disease virus strains with ICPI score of 0.7 lentogenic, while those with a score of more than 1.5 as velogenic. On the other hand, those strains with ICPI scores between 0.7 and 1.5 were mesogenic. For MDT, allantoic fluid with virus was diluted in a series of 10 -fold dilutions with phosphate-buffered saline (PBS) and each dilution inoculated into the allantoic cavity of specific pathogen-free 10-day-old embryonated eggs and incubated at $37^{\circ} \mathrm{C}$. We observed the eggs every 8 hours for 7 days and the time of embryo death was recorded. The MDT was recorded as the mean time (hours) for the minimum lethal dose of the virus to kill all the embryos [1]. We considered isolates with MDT $\leq 60$ hours velogenic: MDT between 61 and 90 hours was mesogenic, while those with MDT $>90$ hours were lentogenic.

2.4. Pathological Characteristics of the NDV Isolates. To determine the pathological characteristics of the NDV isolates, we randomly allocated forty (40) SAN chicken aged four (4) weeks into four experimental groups consisting of three NDV infected groups (group 1-KE001/2015, $n=10$; group 2-KE0698/2016, $n=10$; and group 3-KE0811/2016, $n=10)$ and one control group $(n=10)$. We challenged birds in the NDV infected groups via intraocular inoculation with respective virus suspension $(0.1 \mathrm{ml})$ containing $10^{6} 50 \%$ lethal dose (LD50). We inoculated the control group with $0.1 \mathrm{ml}$ PBS and observed all birds daily for clinical signs of disease. We euthanized and necropsied two birds from each group at day 5 after inoculation and all birds with severe clinical symptoms. We monitored the birds daily until the end of the experiment, two weeks after challenge. Thereafter, we euthanized and necropsied all remaining birds. At necropsy, we collected tissues of trachea, spleen, brain, lung, liver, bursa of Fabricius, proventriculus, and caecal tonsils and fixed them in $10 \%$ buffered formalin. The samples were embedded into the paraffin and sectioned at $3 \mu \mathrm{m}$ and routinely deparaffinized and stained with hematoxylin and eosin for histopathological examination.

2.5. RNA Extraction and Whole-Genome Sequencing. We extracted viral genomic RNA from the allantoic fluid using Trizol reagent (Life Technologies, USA) according to the manufacturer's instructions. The concentration and purity of extracted RNA were measured using a spectrophotometer (ND-1000, Nanodrop Technologies, USA) and Qubit Fluorimeter (Invitrogen, Carlsbad, CA, USA), and the integrity of RNA was visualized by electrophoresis in a $1.2 \%$ formaldehyde agarose gel stained with GelRed and also using the Bioanalyzer. RNA extracts were selected for library preparation when the $260 / 280$ purity index was $\geq 2.0$ and the integral RNA in electrophoresis and Bioanalyzer measurements was greater than eight $(\mathrm{RIN}>8)$. The concentration and purity of the extracted RNA were determined using a Qubit Fluorimeter (Thermo Scientific, Walton, Massachusetts, USA) before storage at $-80^{\circ} \mathrm{C}$ until use.

The nucleotide sequence of the full viral genome was determined using next-generation sequencing (NGS). The cDNA libraries were prepared using a TruSeq RNA sample preparation kit (Illumina, CA, USA). Briefly, mRNA was prepared by purification and fragmentation of $2 \mu \mathrm{g}$ of total RNA using oligo (dT) magnetic beads and was used as a template for cDNA synthesis by random hexamer priming. Paired-end $(2 \times 100$ reads $)$ sequencing was done using Miseq (Illumina). The reads of the full-length viral genome were assembled de novo with SPAdes assembler version 3.10.1 [32]. We confirmed the NGS results with conventional Reverse-Transcription polymerase chain reaction (RT-PCR) using virus-specific primers. This involved purification of amplified products using Qiagen PCR Purification Kit and sequencing purified products by the Sanger method at Macrogen, Korea. The sequences obtained by the Sanger method were $100 \%$ identical to the NGS sequences.

2.6. Phylogenetic Analysis. We obtained complete sequences of reference strains for each NDV genotype from GenBank including the recent Tanzania isolate MK583011 (Supplementary Table S1). We also compared the study isolates with other NDV isolates from Africa, which are present in the GenBank. These sequences from GenBank and the complete coding sequences of the four NDV isolates were aligned using MUSCLE v. 3.8.31 [33, 34]. Phylogenetic and molecular evolutionary analyses were conducted using MEGA (Molecular Evolutionary Genetics Analysis) version 6.0 [35]. We constructed phylogenetic trees using the Maximum Likelihood (ML) method and estimated the tree using bestfit general time-reversible (GTR) model of nucleotide substitution with gamma-distributed rate variation among sites. We employed a bootstrap resampling process (1000 replications) to assess the robustness of individual nodes of phylogeny. Evolutionary distances were computed by the Pairwise Distance method using the Maximum Composite Likelihood method [35].

\section{Results and Discussion}

3.1. Biological Characterization. The three isolated viruses were virulent by the OIE standard criteria. Table 1 shows the MDT in embryonated eggs and ICPI values for the three NDV isolates as well as the amino acid sequence at the cleavage site. The ICPI for the three isolates was $>1.5$, while the MDT was $<60$ hours indicative of velogenic strains. Further, the amino acid sequence motif at the cleavage site of the fusion gene concurred with the ICPI and MDT scores of the three isolates that indicated their velogenic nature.

3.2. Pathological Lesions. All chickens inoculated with NDV study isolates were successfully infected and showed signs of depression, mucoid diarrhea, leg paralysis, comb cyanosis, and head edema and facial swelling with mucoid exudate 
TABLE 1: Results of pathogenicity index tests of three NDV isolates from Kenya.

\begin{tabular}{lccccc}
\hline Strain & Place of origin & Accession number & MDT & ICPI & $\mathrm{F}_{0}$ cleavage site (111-118) \\
\hline KE001/2015 & Makueni & MN685354 & 48.7 & 1.56 & GRRQKR ${ }_{*}$ FV \\
KE0811/2016 & Mombasa & MN685355 & 44.2 & 1.69 & GRRQKR $F V$ \\
KE0698/2016 & Malaba & MN685356 & 38.9 & 1.74 & GRRQKR $_{*}$ FV \\
\hline
\end{tabular}

from the nostrils. Gross lesions consisted of enlargement of organs such as the liver, spleen, and kidneys in the majority of the birds. Gross lesions were also evident in the mucosa of the proventriculus, spleen, liver, and small intestines (Figure 1).

These lesions included hemorrhagic and necrotic patches and blood vessel congestion. The spleen had numerous multifocal whitish spots that were evident on the surface. The trachea also showed mucosal hemorrhages and necrosis with a few birds showing pneumonic consolidation in the lungs with the discharge of caseous exudates from the air sacs. The intestines showed darkened areas of necrosis that were evident on the serosa surface. These necrotic areas in the lumen of the intestines were covered with mucoid content underneath of which were ulcerative foci. The bursa of Fabricius showed marked edema (Figure 1).

All groups infected with the NDV study isolates presented similar histological changes in the multiple organs and tissues at 5 days after challenge. The most extensive damage involved blood vessels and lymphoid tissues. We observed vascular changes including hemorrhages, congestion, and edema in majority of the organs. Severe congestion of blood vessels and diffuse hemorrhages were present in the liver, small intestines, and kidneys.

We observed depletion of the lymphoid tissue in lymphoid organs such as the spleen, bursa, and payers patches. On the other hand, organs such as lungs, small intestines, and liver showed lymphocyte infiltration (Figure 2). The intestinal mucosal epithelium was denuded with loss of villi structure. The findings resemble pathological presentation in infection by highly virulent viruses [36].

3.3. Genome Similarity Analysis. Similarity search of the three isolates on the nucleotide databases through the BLAST algorithm yielded the highest similarity (97\%) to the genome sequence of a previous Kenyan Newcastle disease virus (NDV) isolate, A48 (Accession JQ217420), and Tanzanian isolate, Tanzania/Mbeya/MT15/2012. The isolates also showed high similarity (92\%) to the complete genome sequence Newcastle disease virus strain, Largo/71 from the USA classified as genotype V clade II.

The percentage similarity for nucleotide sequences encoding the different NDV genes was compared between isolate KE001/2015 (Makueni) and other NDV strains including the study strains: KE0811/2016 (Mombasa) and KE0698/2016 (Malaba) and others from the GenBank (NCBI). Study isolates were highly similar to NDV strains grouped under genotype $\mathrm{V}$ ranging from 89 to 97 (Table 2). On the other hand, the similarity of the study isolates to NDV strains of genotypes I and II ranged between 82 and 83 percent. The similarity of the coding regions of KE001/2015
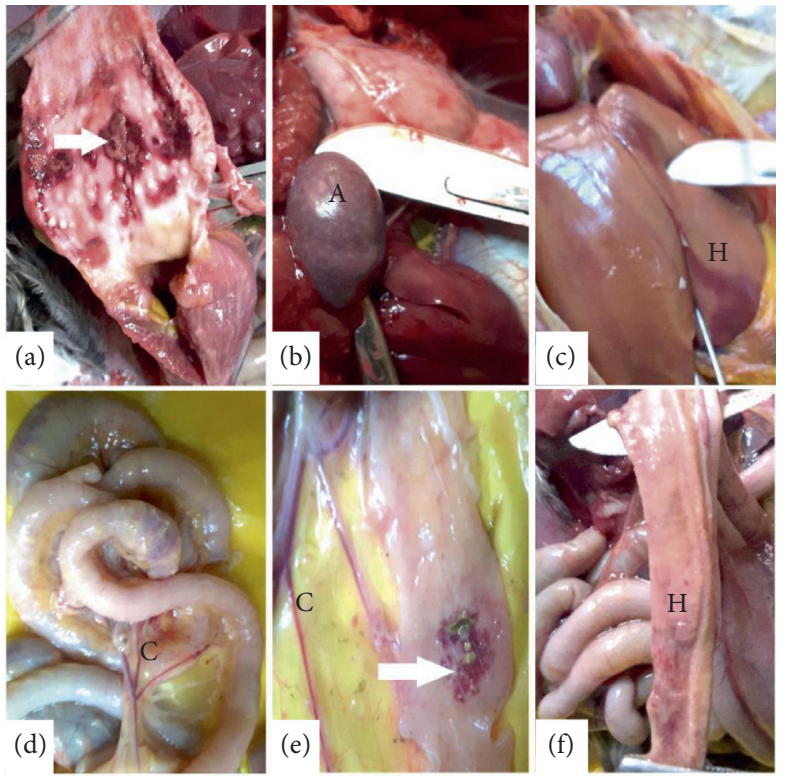

FIGURE 1: Gross pathology lesions observed on organs of specificpathogen-free chicken infected with NDV isolates at 5 days after challenge. First row: (a) proventriculus showed patches of reddened areas on the mucosa (hemorrhagic patches), (b) the spleen was oedematous and showed multifocal white specks (necrotic pointsA), and (c) the liver was oedematous and showed focal reddened hemorrhagic areas $(\mathrm{H})$. Second row: $((\mathrm{d}-\mathrm{f}))$ small intestines showed congestion of blood vessels $(\mathrm{C})$, darkened patches of necrosis (arrow), and diffuse hemorrhages $(\mathrm{H})$ on the mucosa.

(Makueni) to NDV vaccine strains (LaSota, BHG, and I-2) ranged from 77 to $85 \%$, while the similarity to gene sequences of genotype $\mathrm{V}$ strains was the highest ranging from 87 to $98 \%$. We observed the lowest similarity between the study and vaccine strains in the coding sequence of the $\mathrm{V}$ and $\mathrm{P}$ gene.

3.4. Genomic Features of Coding and Noncoding Regions of Isolates. The genomes of the three isolates had a length of 15192 nucleotides with the insertion of six nt (cytosine: “CCCCC (T) C") in the 5' UTR noncoding region of the NP gene between position $1643 \mathrm{nt}$ and $1650 \mathrm{nt}$. The genome of the three isolates was organized as $3^{\prime}$-NP-P-M-F-HN-L-5'. The genomes started with a leader and trailer sequences at the $3^{\prime}$ and $5^{\prime}$ end with lengths of $33055 \mathrm{nt}$ and $114 \mathrm{nt}$, respectively (Table 3 ). Similarly, the isolates had a considerably conserved $10 \mathrm{nt}$ sequence at the start of every gene (GS) and another one at the end (GE) of each gene. The open reading frame of each gene was flanked with $3^{\prime}$ and $5^{\prime}$ end untranslated regions (UTRs) of varying lengths on each end (Table 3). Between GE of one gene and GS of the next gene 


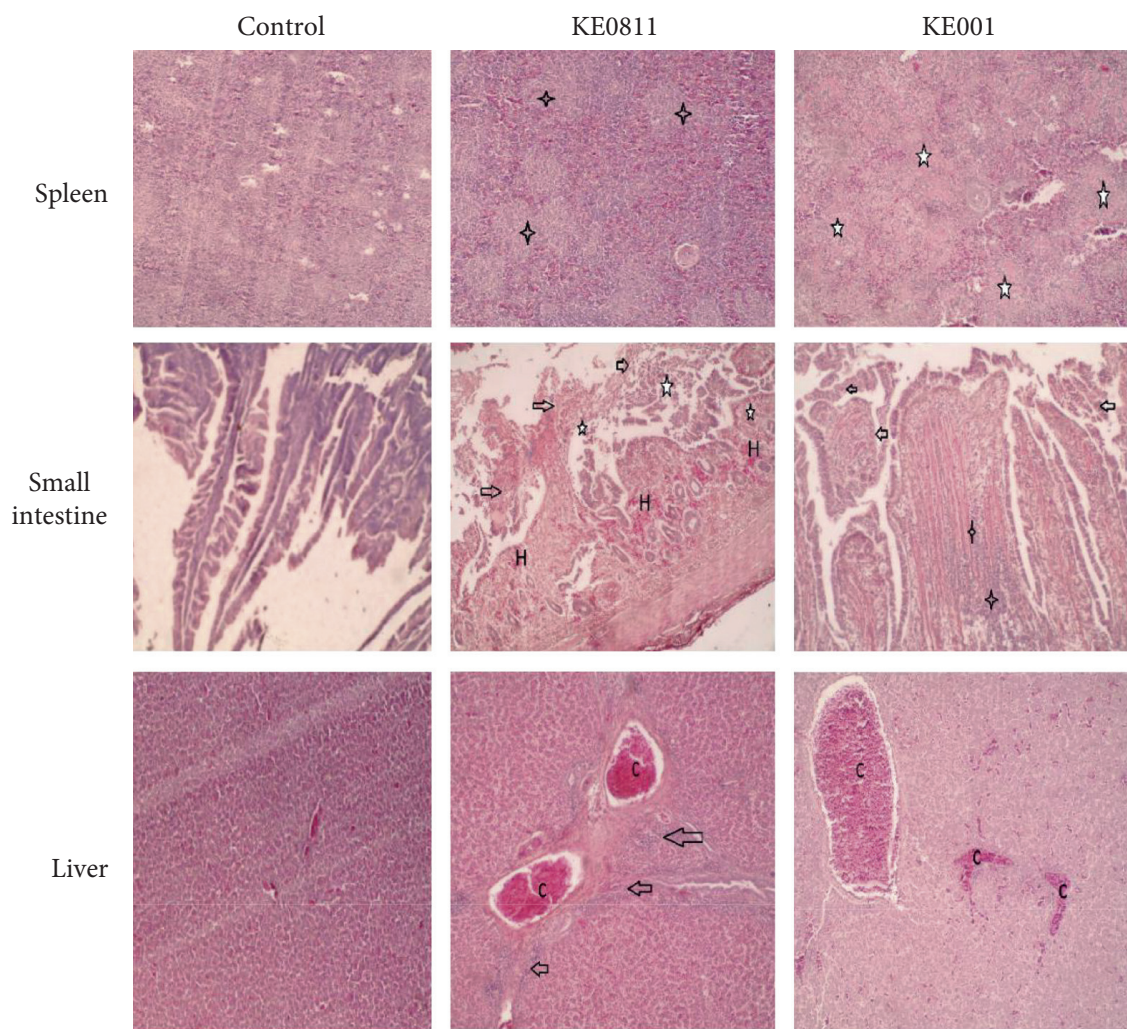

FIGURE 2: Photomicrographs showing hematoxylin and eosin (HE) staining on sections of the spleen, small intestines, and liver at 5 days after challenge. Magnification for panels of spleen and liver and 100x for small intestines $\times 400$. First row: spleen tissue samples from isolate: KE0811 and KE001 showed lymphocyte depletion in the follicles and inflammatory exudates (star). Second row: small intestine tissue samples from NDV challenged groups showed multifocal mucosal hemorrhages (H), lymphocytic infiltration of the submucosa (star), and sloughing of mucosa (arrow). Third row: liver tissue samples of NDV challenged group showed congestion of hepatic blood vessels (C) and lymphocyte infiltration of periportal areas (arrow).

TABLE 2: Percent (\%) similarity of isolate KE001/2015 to nucleotide coding sequences of NDV reference strains.

\begin{tabular}{|c|c|c|c|c|c|c|c|c|}
\hline \multirow{2}{*}{ Strain } & \multirow{2}{*}{ Genome } & \multicolumn{7}{|c|}{ Coding regions } \\
\hline & & $\mathrm{NP}$ & $\mathrm{P}$ & $\mathrm{V}$ & M & $\mathrm{F}$ & $\mathrm{HN}$ & $\mathrm{L}$ \\
\hline KE0811/2016 (Mombasa) & 98.94 & 97.34 & 98.82 & 96.19 & 98.99 & 96.49 & 96.85 & 99.06 \\
\hline KE0698/2016 (Malaba) & 96.63 & 96.8 & 96.54 & 95.87 & 97.35 & 96.18 & 96.73 & 97.0 \\
\hline $\mathrm{A} 48-\mathrm{Vd}$ & 95.61 & 97.82 & - & - & 97.44 & 97.21 & 97.66 & 97.64 \\
\hline Mt15/Tanzania-Vd & 96.76 & 97.75 & 96.46 & 96.50 & 96.80 & 96.61 & 97.02 & 97.56 \\
\hline Largo/71- $V b$ & 92.25 & 93.06 & 90.65 & 90.79 & 91.87 & 91.89 & 92.13 & 93.87 \\
\hline rAnhinga-Va & 89.15 & 90.27 & 87.20 & 87.61 & 91.87 & 89.89 & 89.45 & 91.44 \\
\hline Italien-IV & 86.28 & 88.29 & 84.34 & 83.80 & 86.21 & 87.47 & 87.0 & 88.72 \\
\hline QH1-VIII & 86.49 & 88.23 & 85.10 & 84.12 & 84.01 & 86.02 & 85.95 & 88.90 \\
\hline AV324/96-VI & 85.53 & 87.68 & 82.82 & 81.90 & 86.48 & 86.87 & 86.71 & 87.73 \\
\hline Mukteswar-III & 84.79 & 85.23 & 81.81 & 82.22 & 85.11 & 85.84 & 85.02 & 87.52 \\
\hline ZJ/1/86/Ch-IX & 84.58 & 86.73 & 82.40 & 81.26 & 84.65 & 85.60 & 84.79 & 87.64 \\
\hline Mali/ML007/08-XIV & 84.50 & 85.98 & 83.41 & 80.89 & 84.56 & 84.87 & 84.79 & 87.21 \\
\hline ZA/AL495/04-VII & 84.22 & 85.91 & 81.39 & 83.43 & 85.75 & 85.48 & 86.07 & 87.34 \\
\hline 499-31/2008-XVI & 83.36 & 86.19 & 80.55 & 81.26 & 84.47 & 83.0 & 84.32 & 85.97 \\
\hline MG/1992/08-XI & 82.78 & 84.28 & 80.97 & 78.02 & 83.37 & 82.87 & 83.04 & 84.84 \\
\hline I-2-I & 82.69 & 83.87 & 78.70 & 79.04 & 83.28 & 83.0 & 82.98 & 85.48 \\
\hline BHG-I & 82.32 & 83.46 & 78.70 & 79.68 & 81.73 & 83.24 & 82.69 & 85.30 \\
\hline LaSota-II & 82.12 & 84.14 & 80.30 & 79.68 & 81.91 & 83.36 & 82.68 & 85.10 \\
\hline
\end{tabular}

NP: nucleoprotein; P: phosphoprotein; F: fusion protein; M: matrix protein; HN: hemagglutinin neuraminidase protein; L: RNA-dependent RNA polymerase. 
TABLE 3: Genomic features of three NDV isolates from backyard chicken flocks in Kenya.

\begin{tabular}{|c|c|c|c|c|c|c|c|c|}
\hline \multirow{2}{*}{ ORF } & \multicolumn{5}{|c|}{ Genome characteristics } & \multirow{2}{*}{ IGS } & \multirow{2}{*}{ Gene length } & \multirow{2}{*}{ Protein length } \\
\hline & Gene start & 3UTR & Coding sequence & 5UTR & Gene end & & & \\
\hline NP & $56-65$ & 66 & $122-1591$ & 216 & $1798-1808$ & 1 & 1753 & 489 \\
\hline $\mathrm{P}$ & $1810-1819$ & 73 & $1893-3080$ & 180 & $3250-3260$ & 1 & 1451 & 395 \\
\hline M & $3262-3271$ & 34 & $3296-4390$ & 112 & $4493-4502$ & 1 & 1241 & 364 \\
\hline $\mathrm{F}$ & $4504-4513$ & 46 & $4550-6211$ & 84 & $6285-6295$ & 31 & 1792 & 553 \\
\hline $\mathrm{HN}$ & $6327-6336$ & 91 & $6418-8133$ & 195 & $8318-8328$ & 47 & 2002 & 571 \\
\hline $\mathrm{L}$ & $8376-8386$ & 11 & $8387-15001$ & 77 & $15069-15078$ & NA & 6703 & 2204 \\
\hline Leader & $1-55$ & NA & NA & NA & NA & NA & 55 & NA \\
\hline Trailer & 15079-15192 & NA & NA & NA & NA & NA & 114 & NA \\
\hline
\end{tabular}

NP: nucleoprotein; P: phosphoprotein; F: fusion protein; M: matrix protein; HN: hemagglutinin neuraminidase protein; L: RNA-dependent RNA polymerase; 3UTR: $3^{\prime}$ end untranslated regions (UTRs); 5UTR: $5^{\prime}$ end untranslated regions (UTRs); ORF: open reading frame; IGS: intergenic sequence; 1 : coding sequence including stop codon; 2: number of amino acid bases without stop codon.

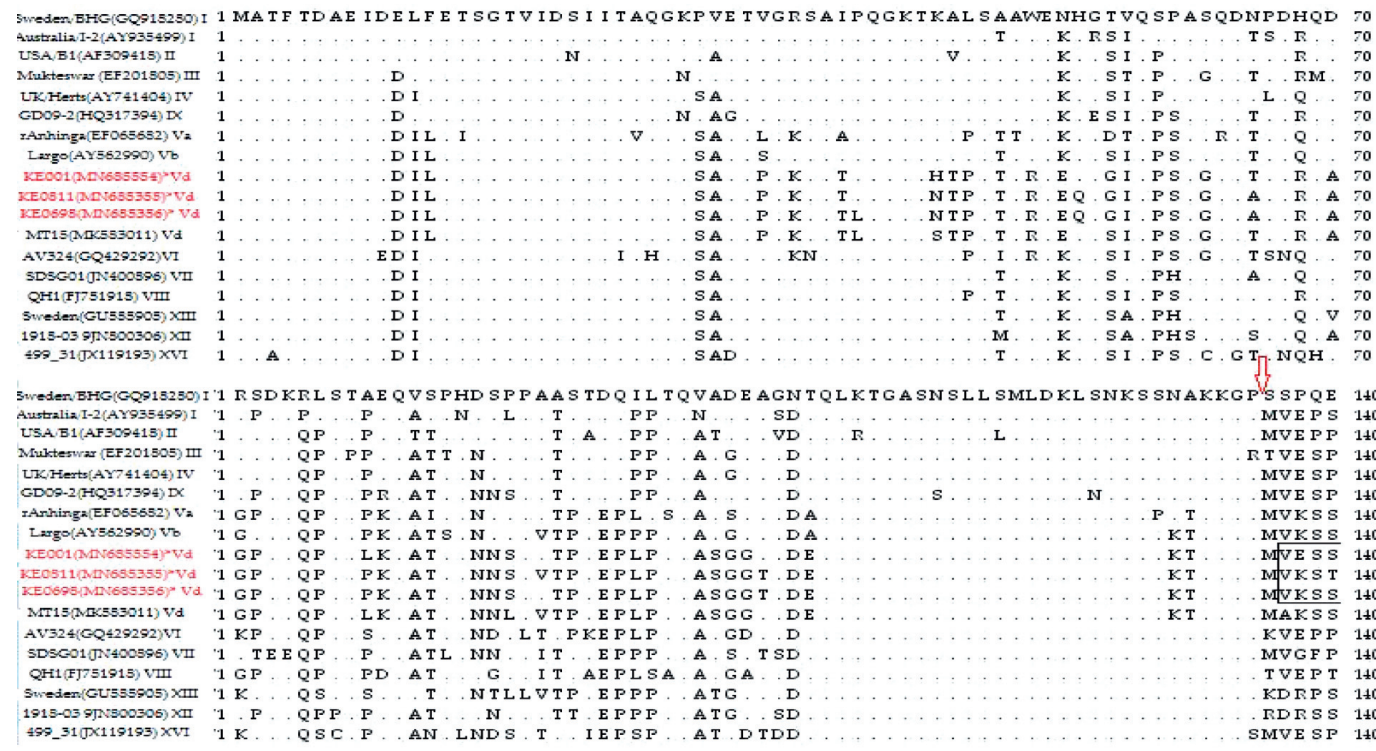
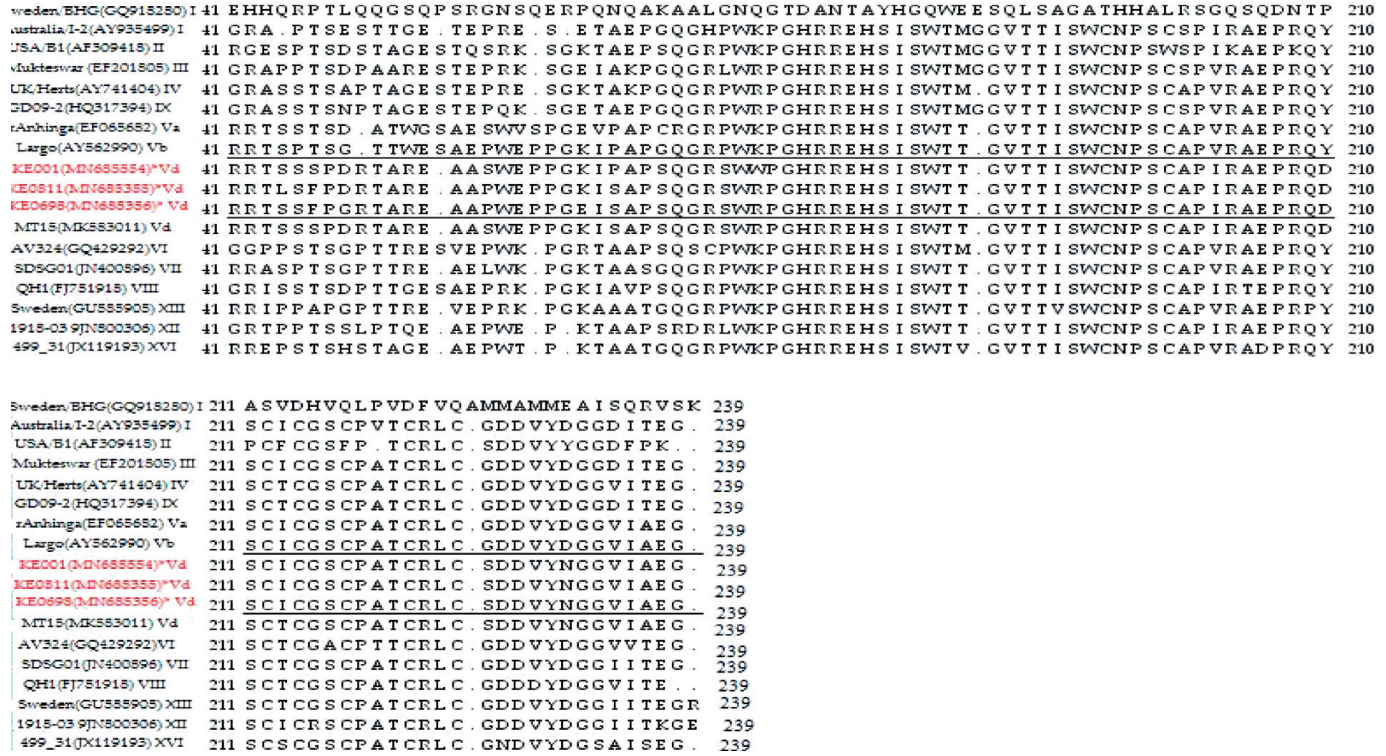

FIgURE 3: Alignment of the $\mathrm{V}$ protein of NDV isolates. The amino acid sequence of the $\mathrm{V}$ protein of the study isolates (highlighted in red) was aligned with NDV V proteins of each genotype reference strain. The P gene-editing site is indicated with an arrow. 
TABLE 4: Comparison of the amino acid residues at the antigenic sites of the hemagglutination neuraminidase protein of the Newcastle disease virus.

\begin{tabular}{|c|c|c|c|c|c|c|c|c|c|c|c|c|c|c|c|c|c|c|c|}
\hline \multirow[b]{3}{*}{ Virus Strain (Accession) } & \multirow[b]{3}{*}{ Genotype } & \multicolumn{18}{|c|}{ Amino acid residues at different antigenic sites } \\
\hline & & \multirow{2}{*}{$\begin{array}{c}1 \\
345\end{array}$} & \multirow{2}{*}{513} & \multicolumn{2}{|c|}{2} & \multicolumn{3}{|c|}{3} & \multicolumn{3}{|c|}{4} & \multicolumn{2}{|c|}{12} & \multicolumn{3}{|c|}{14} & \multicolumn{3}{|c|}{23} \\
\hline & & & & 514 & 521 & 569 & 263 & 287 & 321 & 333 & 356 & 494 & 516 & 347 & 350 & 353 & 193 & 194 & 201 \\
\hline I2 (AY935499) & I & $\mathrm{P}$ & $\mathrm{R}$ & I & S & $\mathrm{D}$ & $\mathrm{N}$ & $\mathrm{D}$ & $\mathrm{R}$ & $\mathrm{K}$ & $\mathrm{K}$ & $\mathrm{D}$ & $\mathrm{R}$ & E & $\mathrm{Y}$ & $\mathrm{R}$ & $\mathrm{L}$ & $S$ & $\mathrm{H}$ \\
\hline Lasota (AF077761) & II & & & & & & & & $\mathrm{K}$ & & & G & & & & & & & \\
\hline Mukteswar (EF201805) & III & & & & & G & $\mathrm{K}$ & & K & $\mathrm{R}$ & & & & & & & & & \\
\hline Herts (AY741404) & IV & & & & & & $S$ & & $\mathrm{~K}$ & $\mathrm{R}$ & & & & & & & & & \\
\hline MT15 (Mk583011) & $\mathrm{Vd}$ & & & & & $\mathrm{N}$ & $\mathrm{R}$ & & $\mathrm{K}$ & & & $\mathrm{N}$ & & & & & & & \\
\hline KE001 (MN685354) & SS & & & & & $\mathrm{N}$ & $\mathrm{R}$ & & K & $\mathrm{R}$ & & $\mathrm{N}$ & & & & & & & \\
\hline KE0811 (MN685355) & SS & & & & & $\mathrm{N}$ & $\mathrm{R}$ & & K & $\mathrm{R}$ & & $\mathrm{N}$ & & & & & & & \\
\hline KE0698 (MN685356) & SS & & & & & $\mathrm{N}$ & $\mathrm{R}$ & & $\mathrm{K}$ & $\mathrm{R}$ & & $\mathrm{N}$ & & $\mathrm{K}$ & & & & & \\
\hline Largo (AY562990) & $\mathrm{Vb}$ & & & & & $\mathrm{N}$ & $\mathrm{K}$ & & $\mathrm{R}$ & $\mathrm{R}$ & & $\mathrm{N}$ & & & & & & & \\
\hline AV324 (GQ429292) & VI & & & $\mathrm{V}$ & & & K & & K & & & & & G & & & & & \\
\hline QH1 (FJ751918) & VIII & & & $\mathrm{M}$ & & & $\mathrm{K}$ & & $\mathrm{D}$ & $\mathrm{R}$ & & & & & & & & & \\
\hline SDSG01 (JN400896) & VII & & & $\mathrm{V}$ & & & K & & $\mathrm{K}$ & & & & & & & & & & \\
\hline GU585905 & XIII & & & $\mathrm{V}$ & & A & $\mathrm{K}$ & & K & & & & Q & & & & & & \\
\hline 1918-03 (JN800306) & XII & & & $\mathrm{V}$ & & & $\mathrm{K}$ & & $\mathrm{K}$ & & & $\mathrm{G}$ & & & & & & & \\
\hline
\end{tabular}

HN antigenic sites $1,2,3,4,12,14$, and 23 shown on the table are essential for receptor recognition. In the table are amino acid residue substitutions at different amino acid positions at the antigenic sites on the sequence of different Newcastle disease virus strains/genotypes compared to that of strain I-2 (AY935499). The amino acid residues shown are at position 345 of antigenic site 1; amino acid at positions 513,514, 521, and 569 at antigenic site 2; amino acid at positions 263 and 287 at antigenic site 3; amino acid at positions 321,333, and 356 at antigenic site 4; amino acid at positions 494 and 516 at antigenic site 12 ; amino acid at positions 347, 350, and 353 at antigenic site 14; amino acid at positions 193, 194, and 201 at antigenic site 23. The universal one-letter codes of the amino acid residues shown are interpreted as follows: P: proline; R: arginine; I: isoleucine; S: serine; D: aspartic acid; N: asparagine; K: lysine; E: glutamic acid; Y: tyrosine; L: leucine; H: histidine; V: valine; W: tryptophan; G: glycine; A: alanine; Q: glutamine. A blank at the residues shows that the sequence of the virus strain has an amino acid similar to that of I-2 Newcastle disease virus strain at that site.

was a conserved sequence, intergenic sequence (IGS). The length of the nucleotide and amino acid sequence of each gene was varied with the RNA-dependent RNA polymerase (L) gene presenting the longest gene.

3.5. Genomic Features of Protein-Coding Regions of Isolates. In multiple sequence alignment, the NP protein of the study isolates showed the highest similarity to NDV strain A48/ Kenya and Mt/Tanzania. The amino acid sequence of the three isolates had three conserved domains on the NP protein: region 1 spanning between aa 171 and 181 with the motif: ${ }^{171}$ QVWVTVAKAMT ${ }^{181}$; region 2 spanning between aa 267 and 277 with the motif: ${ }^{267}$ FFLTLKYGINT ${ }^{277}$; and the third conserved region between aa 322 and 336 with the motif: ${ }^{322}$ FAPAEYAQLYSFAMG ${ }^{336}$. The latter region found in the NP ORF (Open reading frame) of all paramyxoviruses of the genera Avulavirus is a 15 amino acid region and was similarly conserved in all genotype representative strains of NDV used in this study. These regions are important for the formation of the NP-P complexes and regulation of viral replication [37-39].

The phosphoprotein (P) gene had the most variable sequence high variability between the three isolates and other NDV strains but had a conserved site region at nucleotide positions 394-401 (5'-AAAAAGGG-3') corresponding to RNA editing site in the P gene [40]. At this site, insertion of one $G$ gives rise to the $V$ protein, while the addition of two Gs gives rise to $\mathrm{W}$ protein. The $\mathrm{V}$ protein sequence of the study isolates aligned with that of reference genotypes of NDV. The length of the V protein is 239 aa for the NDV strains (Figure 3). The $\mathrm{V}$ protein forms into unique folds through seven cysteine amino acids at the carboxyl- (C-) terminus. For the three isolates, the cysteines are at positions $196,200,212,214,217,221$, and 224 . The V protein of the study isolates was $96.5 \%$ similar to that of Tanzanian isolate: Mbeya/MT15/2012 (MK583011) and 90.8\% similar to strain Largo/71.

The matrix protein of the three isolates had conserved sites among them: ${ }^{40}$ QYRIQRLDSWIDSKE ${ }^{54}$, ${ }^{171}{ }^{1}$ KVNFVSLTVVPRKD ${ }^{185},{ }^{213}$ EVDPRSPLVKSLSRS ${ }^{227}$, ${ }^{247}{ }^{\text {KKGKKVTFDKLERKIRR }}{ }^{263}$, and ${ }^{349}$ KIEKRHTIA$\mathrm{KYNPFK}^{363}$, which were similar in other NDV strains except for a few substitutions. These sites correspond to immunodominant epitopes of the matrix protein [41]. For the $M$ protein to be localized in the nucleus, it utilizes nuclear localization signals (NLS). For the study isolates, the NLS was ${ }^{246}$ DKKGKKVTFDKLERKIRR ${ }^{263}$.

In the fusion protein sequence of the three isolates, the 5 potential asparagine $(\mathrm{N})$ linked glycosylation sites, 85, 191, 366,447 , and 471 , as well as cysteine residue positions, $76,199,338,347,362,370,394,399,401$, and 424 and individual residues, D72, E74, A75, K78, A79, and L343, were highly conserved in the study isolates. Besides, the study strains and other genotypes shared similar neutralizing antibody epitope amino acid residues of the fusion gene at positions 151 to $171,{ }^{151}$ ILRLKESIAATNEAVHEVTDG ${ }^{171}$. The conserved sites have been reported as important functional sites of the F gene $[42,43]$. The key function of the $\mathrm{F}$ protein is to initiate the fusion of viral surface to the host cell membrane. The amino acid motif at the $\mathrm{F}$ gene cleavage site is a major determinant of virulence. For the study 


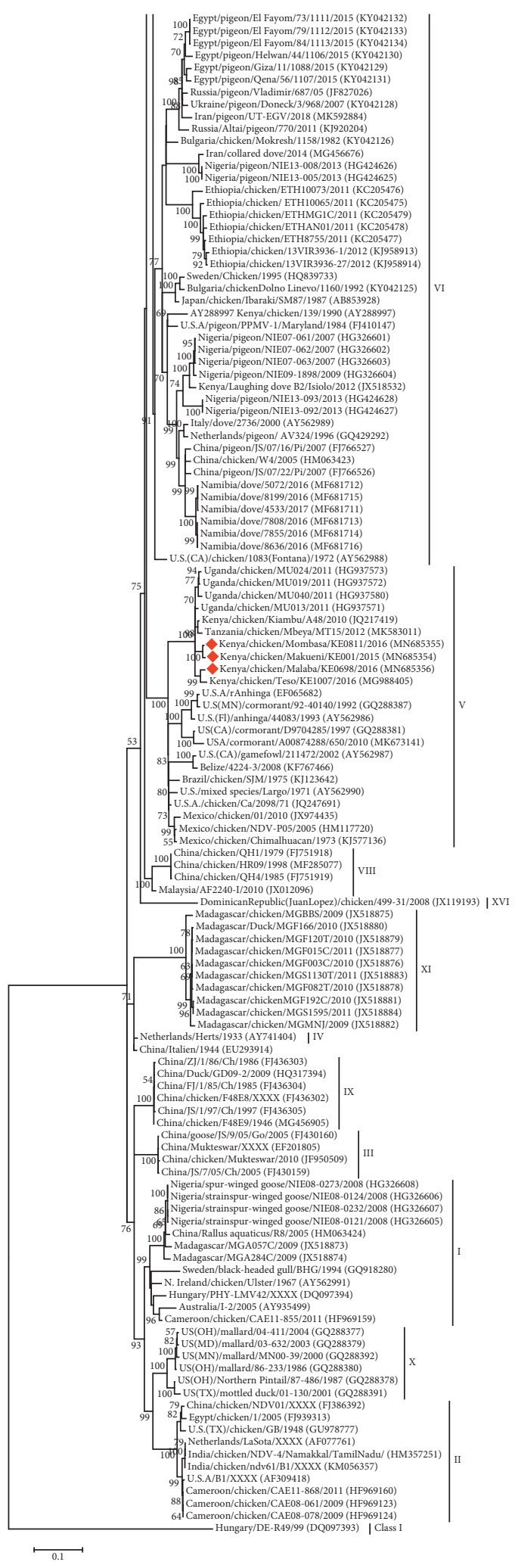

(a)

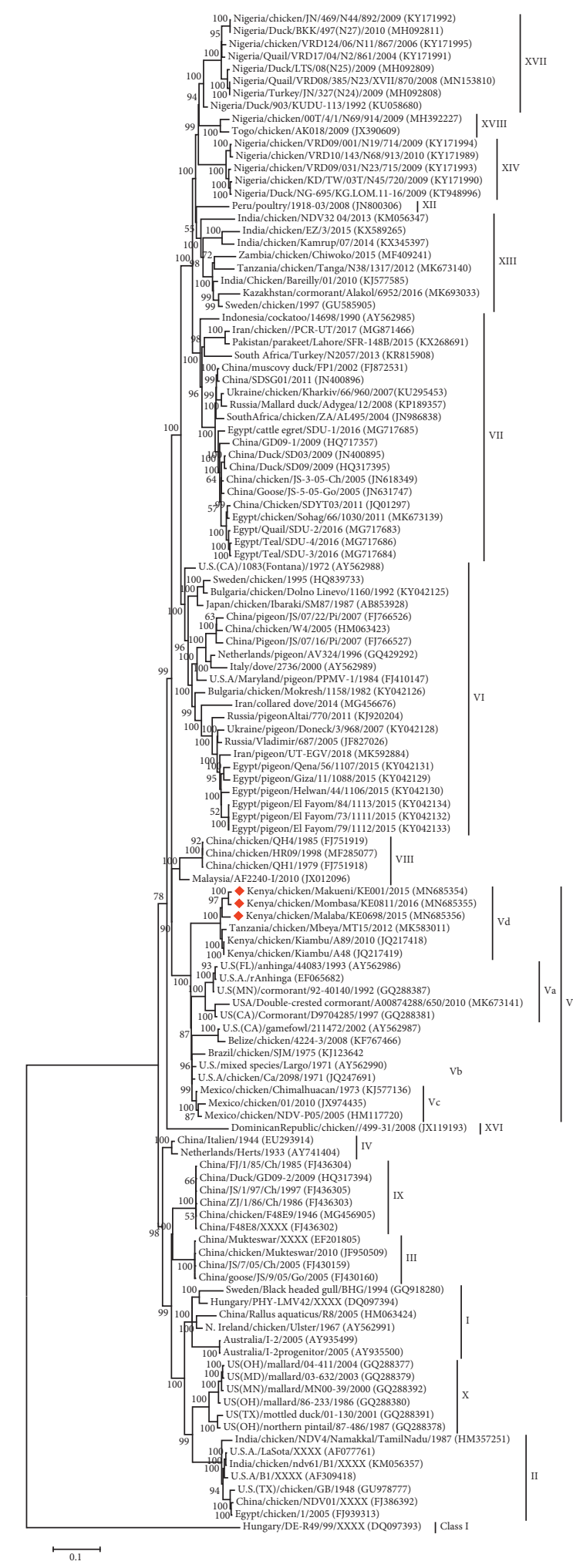

(b)

FIgURe 4: Phylogenetic analysis of NDV complete genome nucleotide sequences. The evolutionary history was inferred by using the Maximum Likelihood method. The percentage of trees in which the associated taxa clustered together based on 1000 replicates is shown next to the branches. (a) The tree was constructed based on 306 nucleotide sequences of the fusion gene with a total of 1662 positions in the final dataset. Highlighted are sequences of isolates from Kenya that were collected in the study. The tree was constructed based on the GTR model with a discrete gamma distribution used to model evolutionary rate differences among sites (2 categories $(+G)$, parameter $=0.33)$ [35]. $(b)$ A phylogenetic tree constructed using 122 nucleotide sequences including most known African isolates. The tree is based on the General Time Reversible (GTR) model with a discrete gamma distribution used to model evolutionary rate differences among sites $(2$ categories $(+G)$, parameter $=0.4054)$. Highlighted are sequences of Kenyan isolates collected in the study. 
isolates, the cleavage site had the motif ${ }^{112} \mathrm{R}-\mathrm{R}-\mathrm{Q}-\mathrm{K}-\mathrm{R}-\mathrm{F}^{117}$ corresponding to the cleavage site of velogenic viruses.

The HN protein plays a critical role in virus infectivity by binding sialic acid-containing receptors. We analyzed the HN protein of study isolates to identify the amino acid residues essential for receptor recognition. Fourteen residues essential for receptor recognition, R174, I175, D198, K236, E258, Y299, Y317, E401, R416, R498, R516, Y526, and E547, in the HN protein of the study isolates were similar to that of other NDV reference strains. To further understand the structure of the HN protein of the study isolates, the seven antigenic sites within the $\mathrm{HN}$ protein involved in the formation of the three-dimensional $\mathrm{HN}$ molecule were compared across the NDV reference strains (Table 4).

Compared to the strains I-2, we observed substitutions at different antigenic sites in the HN protein of the study isolates including D569N at antigenic site 2, N263R at site 3, R321K and $\mathrm{K} 333 \mathrm{R}$ at site 4 , and D494N at site 12 . For the study isolates, the sequence at the antigenic determinant linear epitope of $\mathrm{HN}$ at position 345-353 had the motif ${ }^{345} \mathrm{PDEQDYQVR}^{353}$ with an I/V substitution at aa 352. However, there was a substitution $\mathrm{E} 347 \mathrm{~K}$ for one study isolate, KE0698 (MN685356). Like other NDV strains, the HN protein of study isolates had a conserved amino acid sequence ${ }^{79}$ DVIDRVYKQVALESPLALLSTESIIMNAITSLSYQIN ${ }^{115}$ within the dominant linear antigenic domain of the $\mathrm{HN}$ gene which spans aa 53 to 192 [44]. This was similar in NDV strains except three substitutions at residues V81I, N98S, and T102I observed in the study isolates. The sialic acid binding site of the HN protein [45] of the study isolates, like other NDV strains, was located at aa positions 234-239. For the study isolates, the binding site had the motif ${ }^{234} \mathrm{NRKSCS}^{239}$. Other amino acid residues that form $\mathrm{HN}$ antigen epitopes were similar in the study isolates and other NDV strains including K138, P244, F277, T380, T409, and H482. These sites correspond to the neuraminidase active site that binds the fusion protein [46].

The RNA-dependent RNA polymerase (L) gene of the study isolates and other NDV strains had a highly conserved region between aa 740 and $753\left({ }^{740}\right.$ SHCRVACMVQGDNQ $\left.{ }^{753}\right)$ which corresponds to the putative active site of the $\mathrm{L}$ gene essential to polymerase function [47]. Other conserved domains of the L gene were observed between aa 503 and 607 and 418 between aa 634 and 854 . The L gene protein was highly conserved among the NDV structural 419 proteins in the study isolates.

3.6. Phylogenetic Analysis. Eighteen genotypes (I-XVIII) of NDV class II have been described [5, 17]. Phylogenetic analysis was based on the complete genome sequences including genome sequences of known strains isolated from African countries including South Africa, West Africa, and East Africa (Figure 4). The phylogenetic analysis of complete genome sequences revealed that the study isolates were clustered separately from the sequences from South Africa, which were classified as genotypes VII and XII and those of West Africa that were classified in genotypes XIV and XVIII.
However, the study isolates clustered together with others from East Africa (Tanzanian and previous Kenyan strains) as well as other strains from the United States of America grouped in genotype $\mathrm{V}$ (Figure 4).

Within genotype $\mathrm{V}$, the East African strains including study strains formed a closer cluster that has been referred to as new subgenotype $\mathrm{Vd}$ [5]. The genotype $\mathrm{V}$ of NDV is separated into four subgenotypes $(\mathrm{Va}-\mathrm{d})$. With the earlier classified subgenotypes, $\mathrm{Va}, \mathrm{Vb}$, and $\mathrm{Vc}$ hav been isolated mainly from Europe, Central America, and South America $[9,48,49]$. To confirm the results obtained by genome analysis, we performed a phylogenetic analysis based on the complete $\mathrm{F}$ gene coding sequence, which incorporated more sequences representing the known subgenotypes of genotype V (Figure 4(a)).

\section{Conclusion}

Complete genome, protein level, and biological analysis of three NDV isolates from backyard chicken in Kenya demonstrated that the isolates have the biological and pathological characteristics of virulent viruses. Further, the study demonstrated that the isolates belong to genotype $\mathrm{V}$ and subgenotype $\mathrm{Vd}$. This is, therefore, the first genomic and pathological characterization of NDV of subgenotype Vd. Subgenotype Vd seems to be responsible for current outbreaks in Kenya and parts of the East African region. NDV isolates, sequences, and findings obtained from the study will, therefore, be valuable in analyzing the nature and diversity of NDV in the East African region. The study findings contribute to the currently available genome data on NDV in Africa and worldwide. Through this work, we also establish local and regional reference viruses for future studies in the development of improved control and diagnostic strategies.

\section{Data Availability}

The full-genome sequence of the NDV isolates analyzed in this study has been submitted to GenBank under Accession nos MN685354, MN685355, and MN685356.

\section{Conflicts of Interest}

The authors declare that there are no conflicts of interest regarding the publication of this paper.

\section{Acknowledgments}

This research work was supported by the International Foundation for Science (IFS), Stockholm Sweden through Research Grant 13_B/32827. The authors acknowledge the International Foundation for Science (IFS), Stockholm, Sweden, for financial support. The authors would also like to thank Moses Ogugo, Samuel Imujaro, and John Odera for their support in the field sampling and laboratory analysis. The authors also acknowledge the Country Livestock and Veterinary field staff for their technical support. 


\section{Supplementary Materials}

Table S1 describes the accession numbers, strain name, year, and country of isolation of reference sequences used for comparison with study sequences. The references are of known genotypes of Newcastle disease virus and are available in the GenBank including those isolated from Africa. Figure S2 shows the whole phylogenetic tree representing full coding sequences of the fusion gene of 306 strains of NDV available in the GenBank including sequences obtained from this study and all known isolates from Africa. The fusion gene is useful in the characterization of NDV. Therefore, this Maximum Likelihood tree is useful for comparison with the complete genome phylogenetic tree that is presented in Figure 4. The two trees are similar in their characterization of study isolates. (Supplementary Materials)

\section{References}

[1] OIE, "Newcastle disease: general infectious disease sheets," 2015, https://www.oie.int/fileadmin/Home/eng/Media_Center/ docs/pdf/Disease_cards/NEWCAS-EN.pdf.

[2] A. Ashraf and M. S. Shah, "Newcastle disease: present status and future challenges for developing countries," African Journal of Microbiology Research, vol. 8, no. 5, pp. 411-416, 2014.

[3] G. K. Amarasinghe, Y. Bào, C. F. Basler et al., "Taxonomy of the order Mononegavirales: update 2017," Archives of Virology, vol. 162, no. 8, pp. 2493-2504, 2017.

[4] P. Gogoi, K. Ganar, and S. Kumar, "Avian paramyxovirus: a brief review," Transboundary and Emerging Diseases, vol. 64, no. 1, 2017.

[5] K. M. Dimitrov, A. M. Ramey, X. Qiu, J. Bahl, and C. L. Afonso, "Temporal, geographic, and host distribution of avian paramyxovirus 1 (Newcastle disease virus)," Infection, Genetics and Evolution, vol. 39, pp. 22-34, 2016.

[6] K. Ganar, M. Das, S. Sinha, and S. Kumar, "Newcastle disease virus: current status and our understanding," Virus Research, vol. 184, pp. 71-81, 2014.

[7] D. G. Diel, L. H. A. da Silva, H. Liu, Z. Wang, P. J. Miller, and C. L. Afonso, "Genetic diversity of avian paramyxovirus type 1: proposal for a unified nomenclature and classification system of Newcastle disease virus genotypes," Infection, Genetics and Evolution, vol. 12, no. 8, pp. 1770-1779, 2012.

[8] R. S. d. Almeida, S. Hammoumi, P. Gil et al., "New avian paramyxoviruses type I strains identified in Africa provide new outcomes for phylogeny reconstruction and genotype classification," PLoS One, vol. 8, no. 10, Article ID e76413, 2013.

[9] L. Susta, K. R. Hamal, P. J. Miller et al., "Separate evolution of virulent newcastle disease viruses from Mexico and Central America," Journal of Clinical Microbiology, vol. 52, no. 5, pp. 1382-1390, 2014.

[10] S. C. Courtney, L. Susta, D. Gomez et al., "Highly divergent virulent isolates of Newcastle disease virus from the Dominican Republic are members of a new genotype that may have evolved unnoticed for over 2 decades," Journal of Clinical Microbiology, vol. 51, no. 2, pp. 508-517, 2013.

[11] A. Czeglédi, D. Ujvári, E. Somogyi, E. Wehmann, O. Werner, and B. Lomniczi, "Third genome size category of avian paramyxovirus serotype 1 (Newcastle disease virus) and evolutionary implications," Virus Research, vol. 120, no. 1-2, pp. 36-48, 2006.
[12] C. J. Snoeck, A. A. Owoade, E. Couacy-Hymann et al., "High genetic diversity of Newcastle disease virus in poultry in west and central Africa: cocirculation of genotype XIV and newly defined genotypes XVII and XVIII," Journal of Clinical Microbiology, vol. 51, no. 7, pp. 2250-2260, 2013.

[13] C. Abolnik, C. Mubamba, D. B. R. Wandrag et al., "Tracing the origins of genotype VII h Newcastle disease in southern Africa," Transboundary and Emerging Diseases, vol. 65, no. 2, pp. e393-e403, 2018.

[14] U. Molini, G. Aikukutu, S. Khaiseb, G. Cattoli, and W. G. Dundon, "First genetic characterization of newcastle disease viruses from Namibia: identification of a novel VIIk subgenotype," Archives of Virology, vol. 162, no. 8, pp. 2427-2431, 2017.

[15] C. Abolnik, C. Mubamba, G. Dautu, and B. Gummow, "Complete genome sequence of a newcastle disease genotype XIII virus isolated from indigenous chickens in Zambia," Genome Announcements, vol. 5, no. 34, pp. e00841-e00917, 2017.

[16] C. J. Snoeck, A. T. Adeyanju, A. A. Owoade et al., "Genetic diversity of Newcastle disease virus in wild birds and pigeons in West Africa," Applied and Environmental Microbiology, vol. 79, no. 24, pp. 7867-7874, 2013.

[17] L. Susta, M. E. B. Jones, G. Cattoli et al., "Pathologic characterization of genotypes XIV and XVII newcastle disease viruses and efficacy of classical vaccination on specific pathogen-free birds," Veterinary Pathology, vol. 52, no. 1, pp. 120-131, 2015.

[18] G. Cattoli, A. Fusaro, I. Monne et al., "Emergence of a new genetic lineage of Newcastle disease virus in west and central Africa-implications for diagnosis and control," Veterinary Microbiology, vol. 142, no. 3-4, pp. 168-176, 2010.

[19] D. Mulisa, M. W/Kiros, R. Alemu et al., "Characterization of Newcastle disease virus and poultry-handling practices in live poultry markets, Ethiopia," Springerplus, vol. 3, no. 1, p. 459, 2014.

[20] D. Damena, A. Fusaro, M. Sombo et al., "Characterization of Newcastle disease virus isolates obtained from outbreak cases in commercial chickens and wild pigeons in Ethiopia," Springerplus, vol. 5, no. 1, p. 476, 2016.

[21] H. Chaka, F. Goutard, P. Gil et al., "Serological and molecular investigation of Newcastle disease in household chicken flocks and associated markets in Eastern Shewa zone, Ethiopia," Tropical Animal Health and Production, vol. 45, no. 3, pp. 705-714, 2013.

[22] M. G. Yongolo, H. Christensen, K. Handberg, U. Minga, and J. E. Olsen, "On the origin and diversity of Newcastle disease virus in Tanzania," Onderstepoort Journal of Veterinary Research, vol. 78, no. 1, 2011.

[23] N. Elmardi, M. Bakheit, and A. Khalafalla, "Phylogenetic analysis of some Newcastle disease virus isolates from the Sudan," Open Veterinary Journal, vol. 6, no. 2, pp. 89-97, 2016.

[24] W. Hassan, S. A. M. Khair, B. Mochotlhoane, and C. Abolnik, "Newcastle disease outbreaks in the Sudan from 2003 to 2006 were caused by viruses of genotype 5d," Virus Genes, vol. 40, no. 1, pp. 106-110, 2010.

[25] M. O. Otim, H. Christensen, P. H. Jorgensen, K. J. Handberg, and M. Bisgaard, "Molecular characterization and phylogenetic study of Newcastle disease virus isolates from recent outbreaks in eastern Uganda," Journal of Clinical Microbiology, vol. 42, no. 6, pp. 2802-2805, 2004.

[26] D. K. Byarugaba, K. K. Mugimba, J. B. Omony et al., "High pathogenicity and low genetic evolution of avian 
paramyxovirus type I (Newcastle disease virus) isolated from live bird markets in Uganda," Virology Journal, vol. 11, no. 1, p. 173, 2014.

[27] R. Servan de Almeida, O. F. Maminiaina, P. Gil et al., "Africa, a reservoir of new virulent strains of Newcastle disease virus?" Vaccine, vol. 27, no. 24, pp. 3127-3129, 2009.

[28] A. Conan, F. L. Goutard, S. Sorn, and S. Vong, "Biosecurity measures for backyard poultry in developing countries: a systematic review," BMC Veterinary Research, vol. 8, no. 1, p. 240, 2012.

[29] A. H. Jibril, J. U. Umoh, J. Kabir et al., "Newcastle disease in local chickens of live bird markets and households in Zamfara state, Nigeria," ISRN Epidemiology, vol. 2014, Article ID 513961, 4 pages, 2014.

[30] I. N. Ogali, L. W. Wamuyu, J. K. Lichoti, E. O. Mungube, B. Agwanda, and S. C. Ommeh, "Molecular characterization of Newcastle disease virus from backyard poultry farms and live bird markets in Kenya," International Journal of Microbiology, vol. 2018, Article ID 2368597, 11 pages, 2018.

[31] Federation of Animal Science Societies, "Guide for the care and use of agricultural animals in research and teaching," January 2010, https://www.asas.org/docs/default-source/ default-document-library/ag_guide_3rded.pdf?sfvrsn=4.

[32] A. Bankevich, S. Nurk, D. Antipov et al., "SPAdes: a new genome assembly algorithm and its applications to single-cell sequencing," Journal of Computational Biology, vol. 19, no. 5, pp. 455-477, 2012.

[33] R. C. Edgar and S. Batzoglou, "Multiple sequence alignment," Current Opinion in Structural Biology, vol. 16, no. 3, pp. $368-373,2006$.

[34] R. C. Edgar, "MUSCLE: multiple sequence alignment with high accuracy and high throughput," Nucleic Acids Research, vol. 32, no. 5, pp. 1792-1797, 2004.

[35] K. Tamura, G. Stecher, D. Peterson, A. Filipski, and S. Kumar, "MEGA6: molecular evolutionary genetics analysis version 6.0," Molecular Biology and Evolution, vol. 30, no. 12, pp. 2725-2729, 2013.

[36] D. G. Diel, L. Susta, S. Cardenas Garcia et al., "Complete genome and clinicopathological characterization of a virulent Newcastle disease virus isolate from South America," Journal of Clinical Microbiology, vol. 50, no. 2, pp. 378-387, 2012.

[37] R. E. Randall and A. Bermingham, "NP:P and NP:V interactions of the paramyxovirus simian virus 5 examined using a novel protein:protein capture assay," Virology, vol. 224, no. 1, pp. 121-129, 1996.

[38] S. M. Horikami, S. Smallwood, and S. A. Moyer, "The Sendai virus $\mathrm{V}$ protein interacts with the NP protein to regulate viral genome RNA replication," Virology, vol. 222, no. 2, pp. 383-390, 1996.

[39] S. Longhi, "Nucleocapsid structure and function," Current Topics in Microbiology and Immunology, Springer, vol. 329, pp. 103-128, , Berlin, Germany, 2009.

[40] M. Steward, I. B. Vipond, N. S. Millar, and P. T. Emmerson, "RNA editing in Newcastle disease virus," Journal of General Virology, vol. 74, no. 12, pp. 2539-2547, 1993.

[41] F. El Najjar, A. Schmitt, and R. Dutch, "Paramyxovirus glycoprotein incorporation, assembly and budding: a three way dance for infectious particle production," Viruses, vol. 6, no. 8, pp. 3019-3054, 2014.

[42] S. Seth, A. L. Goodman, and R. W. Compans, "Mutations in multiple domains activate paramyxovirus $\mathrm{F}$ protein-induced fusion," Journal of Virology, vol. 78, no. 16, pp. 8513-8523, 2004.
[43] S. Akhtar, M. A. Muneer, K. Muhammad et al., "Molecular characterization and epitope mapping of fusion (F) and hemagglutinin $(\mathrm{HN})$ genes of avian paramyxovirus serotype $\mathrm{i}$ from peacocks in Pakistan," Pakistan Journal of Zoology, vol. 49, no. 2, pp. 755-759, 2017.

[44] P. Yuan, K. A. Swanson, G. P. Leser, R. G. Paterson, R. A. Lamb, and T. S. Jardetzky, "Structure of the Newcastle disease virus hemagglutinin-neuraminidase (HN) ectodomain reveals a four-helix bundle stalk," Proceedings of the National Academy of Sciences, vol. 108, no. 36, pp. 1492014925, 2011.

[45] S. Crennell, T. Takimoto, A. Portner, and G. Taylor, "Crystal structure of the multifunctional paramyxovirus hemagglutinin-neuraminidase," Nature Structural Biology, vol. 7, no. 11, pp. 1068-1074, 2000.

[46] D. E. Anderson and L. Wang, The Biology of Paramyxoviruses, Caister Academic Press, Norfolk, UK, 2015.

[47] S. N. Rout and S. K. Samal, "The large polymerase protein is associated with the virulence of Newcastle disease virus," Journal of Virology, vol. 82, no. 16, pp. 7828-7836, 2008.

[48] D. G. Diel, P. J. Miller, P. C. Wolf et al., "Characterization of Newcastle disease viruses isolated from cormorant and gull species in the United States in 2010," Avian Diseases, vol. 56, no. 1, pp. 128-133, 2012.

[49] E. Wehmann, D. Ujvári, H. Mazija et al., "Genetic analysis of Newcastle disease virus strains isolated in Bosnia-Herzegovina, Croatia, Slovenia and Yugoslavia, reveals the presence of only a single genotype, V, between 1979 and 2002," Veterinary Microbiology, vol. 94, no. 4, pp. 269-281, 2003. 